\title{
III CONGRESO NACIONAL DE ALIANZA POPULAR
}

Los días 14, 15 y 16 de diciembre de 1979 tuvo lugar en Madrid el III Congreso Nacional de Alianza Popular con asistencia de mil doscientos noventa y un delegados de todas las provincias españolas.

El Congreso se celebraba después que el partido atravesara una situación de cierta conflictividad cuyos resultados fueron la dimisión de su presidente, Félix Pastor, y los vicepresidentes, Isabel Barroso y Carlos Arges. La presidencia fue ocupada de manera interina, hasta la celebración del Congreso, por Fraga Iribarne.

El tema que ha suscitado mayores controversias antes y durante el Congreso fue la modificación de los estatutos. Las modificaciones presentadas por la ponencia dan al partido un carácter fuertemente presidencialista. La enmienda a la totalidad presentada por Guillermo Piera - basada en lo que consideraba confusión entre liderazgo y omnipresencia y al que se consideraba ligado al presidente y vicepresidentes que con anterioridad al Congreso habían presentado su dimisión- sólo obtuvo 32 votos a favor y 19 abstenciones.

La ponencia política defendida por Fraga fue aprobada con 24 votos en contra. Una candidatura única y cerrada se presentó a los compromisarios. Votaron 947 de los 1.200 asistentes, de los que 875 dieron su respaldo al Comité Ejecutivo Nacional propuesto y 71 votaron en blanco.

Por último, por decisión del III Congreso Nacional de Alianza Popular (Partido Unido y Federación), se deja sin efecto la federación con Unión Nacional Española y Acción Democrática Española y se acuerda la federación de los partidos Acción por Ceuta y Unión Liberal, Popular y Democrática de Ibiza y Formentera (S'Unio).

El Comité Ejecutivo de la Federación de Alianza Popular está compuesto por las mismas personas que el Comité del Partido Unido Alianza Popular, excepción hecha de los vicepresidentes, cargos para los que fueron elegidos don Alvaro Lapuerta Quinteros, don Abel Matutes Juan y don Juan Montesinos García. 


\section{COMITE EJECUTIVO DEL PARTIDO UNIDO DE ALIANZA POPULAR}

Presidente:

D. Manuel Fraga Iribarne.

Vicepresidentes:

D. José María Ruiz Gallardón.

D. Luis Ortiz Alvarez.

D. Manuel García Amigo.

Secretario general:

D. Jorge Verstrynge Rojas.

Tesorero nacional:

Doña Begoña Urquijo.

Secretarios generales adjuntos:

D. Guillermo Kirkpatric Mendaro.

D. Jesús Pérez Bilbao.

D. Miguel Ramírez González.
Vocales nacionales:

D. José Manuel González Páramo.

Doña Carmen Llorca Villaplana.

D. Antonio Hernández Mancha

D. Rodrigo Rato Figaredo.

D. Manuel Gasset Dorado.

Representante nacional de Nuevas Generaciones:

D. Alejandro Francisco Martín Carrero.

Representante de los Senadores:

D. Carlos Pinilla Touriño.

Representante de los Diputados:

D. Juan Luis de la Vallina Velarde.

\section{FRAGMENTO DEL DISCURSO LEIDO AL CONGRESO POR MANUEL FRAGA}

\section{ALGUNAS IDEAS PARA LA RECONSTRUCCION DE UNA ESPAÑA CON FUTURO}

Digo que vamos a hablar de ideas. No basta, en política, con sentimientos, por nobles que sean. Si el corazón tiene sus razones, que la cabeza no comprende, también el cerebro tiene su manera de amar, como acaso no sabe el corazón. Cuanto más movidas se ponen las cosas, y más calientes los hechos, más hace falta utilizar el frío bisturí de la razón.

Ideas, digo, por otra parte, y no ideología; no pretendemos ponerle al mundo y a la vida un corsé ideológico; no creemos, como los marxistas, que se pueda reducir la sociedad de los hombres, seres libres, a una sola clave, ni a un dogma estancado. Lo que pretendemos es basar nuestra acción en un conjunto de principios, de verdades sólidas, de juicios realistas, con los que se pueda fijar un criterio ante cada nueva situación. Ya el viejo Metternich le dijo al entonces joven Donoso Cortés, en 1848 , que un sistema de principios será siempre superior a un programa ideológico, como un cañón giratorio lo es a uno fijo.

Todos sabemos lo que quiere decir, a su vez, tener o no tener principios, como verdades que rigen una vida, una acción, una moral. También sabemos que no se puede tener moral, en el sentido de valor decidido, si no se defiende de verdad lo que uno reconoce como la verdad. Frente a esto está la actitud oportunista. El oportunismo, en todas sus formas y manifestaciones, consiste en hacer las cosas no por lo que valen, sino por lo que rentan; no por el fondo de las cuestiones, sino por la imagen que proyecta la acción. A propósito de la famosa imagen, que hoy 
parece ser el criterio único de ciertos políticos, opino como el senador Fulbright, que nada tiene de malo para un gobernante el dar buena imagen en televisión; pero que es anómalo no tener en cuenta que son condiciones mucho más importantes la inteligencia, el carácter y la decisión; y, por encima de todo, el tener principios.

Reconstrucción: que no es, en este momento, desgraciadamente, una palabra excesiva. Porque España está hoy siendo destruida, y el trabajo de la piqueta demoledora continúa.

La Política (con mayúscula) es justamente arquitectura, como dijo Aristóteles; es construcción de organizaciones válidas en el disperso mundo de lo social. En política es válida también la frase de Valéry, refiriéndose al mundo de las artes: sólo la arquitectura crea espacios sólidos en los cuales puedan asentarse las demás creaciones del espítitu.

La reconstrucción de una sociedad en crisis plantea problemas muy complejos. En primer lugar, de lenguaje: es por la corrupción del lenguaje por donde se manifiesta antes una crisis de Estado. Las palabras se cargan de intencionalidad política: es «progresivo» lo que uno quiere, «regresivo» o «nostálgico» lo que pretende el otro; el que hace violencia a la ley y a los demás es «revolucionario» si es de uno, y «terrorista» si es del otro, y así sucesivamente.

En segundo lugar, requiere un serio análisis de situación, una comprensión del mundo en que vivimos. Los que están destruyendo a España no sólo vuelven a reproducir los mismos errores de 1812, de 1869, de 1931, sino que quieren ignorar el mundo en que vivimos, un mundo ya de vuelta de muchas utopías que entre nosotros renacen.

En tercer lugar, hay que establecer buenos cimientos: toda construcción política hecha a la ligera, sin que fragüen sus fundamentos y que no se presuponga la seguridad interior y exterior, cae al primer soplo del viento.

En cuarto lugar, hace falta la capacidad y buena distribución del edificio que permita la posibilidad para el mayor número. Pero nunca se puede lograr un consenso al cien por cien, y buscarlo es echarse en manos de los más violentos; toda sociedad tiene delincuentes y terroristas que poner a raya.

$Y$, en fin, hay un problema de forma o de estilo. Una sociedad en forma no es lo mismo que una sociedad acicalada; no es lo mismo construir un Escorial para la eternidad que una falla para que arda después de unas fiestas.

Futuro: porque justamente la política es una visión del futuro, y cada vez más, porque las decisiones sociales se toman cada vez a más largo plazo, y porque cada decisión compromete, cada vez, sectores más importantes de la evolución futura de las sociedades y del destino personal de sus miembros. Pensemos en la trascendencia futura de lo que en estas mismas semanas se está decidiendo sobre autonomías regionales, o en lo que supondrá, dentro de veinte años, el tomar o no a tiempo el tren de las nuevas formas de energía. En este sentido, toda política miope, pensando sólo en el corto plazo, es por definición antipolítica; es la negación de una verdadera política, en el sentido noble y profundo de esta palabra.

Ahora bien, en este momento la cuestión del futuro de España es aún de mayor enjundia y trascendencia. Porque está en discusión la misma unidad e identidad de la nación, y por lo mismo la propia subsistencia de España como Estado nacional.

En cada fin de siglo se viene planteando para España esta grave cuestión: en 1700, al final de la dinastía de los Austrias, y la apertura de la Guerra de Sucesión, en la que perdimos nuestros territorios italianos y en los Países Bajos e incluso Mahón y Gibraltar; en el primer cuarto del siglo pasado, con el fin de la monarquía absoluta, el inicio de los tiempos nuevos en las Cortes de Cádiz, la Guetra de la Independencia y la pérdida del imperio americano; en el 98, cuando un Ganivet pudo hablar del «finis hispaniae»; en los años treinta, en los que nos enfrentamos a muerte en una nueva guerra civil. 
El fin de las naciones no es una mera cuestión académica; la Hispania es un gigantesco cementerio de organizaciones políticas periclitadas. $\mathrm{Y}$ no vale decir que siempre subsiste algo: ni Egipto, ni Grecia, ni Italia son los mismos de antes. España se fue haciendo, sobre las tribus primitivas, primero por la romanización y la cristianización, que le dieron una cultura común; después por la unificación política y jurídica del reino visigodo; más tarde, por la empresa común de la Reconquista; proceso que culmina en la reunificación política bajo los Reyes Católicos, y las nuevas empresas de creación de una cultura hispánica, a nivel mundial; y un proceso de centralización y cooperación, impulsado por el despotismo ilustrado del siglo xvIII y el liberalismo en el siglo XIX.

Este proceso está en desgraciada involución desde hace un siglo. La primera República hizo un primer intento de federalizar a España, que terminó en la tragicomedia del cantonalismo. La segunda guerra carlista, a diferencia de la primera, que se había hecho sobre una idea de España y su tradición, exacerbó los temas foralistas, y fue de ciertos grupos carlistas de donde surgió el mayor impulso para los nuevos nacionalismos catalán y vasco, que envenenarán la vida política de primeros de este siglo. La segunda República, al lado de la crisis económica y el maximalismo revolucionario de anarquistas, socialistas y comunistas, tuvo como una de las causas más graves de su fracaso en la insolidaridad de los nacionalismos catalanes y vascos, como se vio en octubre de 1931 y de 1936 a 1939, siendo a este respecto irrefutable el testimonio reiterado de Manuel Azaña. Hoy España, su bandera, su lengua, su Derecho, están en clara contestación, y la guerra revolucionaria y separatista están a la orden del día, mientras que la absurda idea de «nacionalidades» y la descomposición del sistema educativo español preparan las más graves cuestiones para un futuro muy próximo. Y en fin, España. España es para Alianza Popular lo único importante. Como movimiento político, existimos por España y para España. Queremos una España moderna, con libertades, con autonomías, con democracia, con tolerancia, con apertura al exterior, con todo lo bueno y lo deseable, pero que sea España, y no un guiñapo, desunida, destrozada, empobrecida y, en definitiva, disuelta, como tantas otras sociedades que hoy se encuentran tristemente en quiebra.

No supone esto querer imponer una idea rígida de lo que deba ser España, ni menos contraponer España a los españoles. Se nos ha acusado de preocuparnos menos de la suerte de éstos, como personas en busca de su propia felicidad y más de una España institucional, concebida de modo abstracto e idealista; y a través de ello, favorecer a los más favorecidos por el sistema social y a sus dirigentes de siempre. Todo lo contrario: lo que pensamos es que los españoles no pueden salvarse como versos sueltos, ni por regiones, o por clases sociales, o por sexos, o por grupos de edad; lo tienen que hacer juntos, como tales españoles. El mejor dividendo del patriotismo de franceses y alemanes, de ingleses o japoneses, es para ellos mismos, por tener todas las ventajas de una sociedad fuerte, rica y respetada.

Lo increíble es que nadie pueda pensar que en esto España pueda ser diferente. Por eso, repito, nosotros queremos España con todo lo bueno y todo lo moderno; con todo lo que se lleve por ahí, y sea progresivo y atractivo; pero España sin dudas ni vacilaciones.

Tampoco estamos (repito igualmente) por una España como ya fue, incluso en sus mejores días; las naciones, como los tíos, no vuelven hacia atrás. España nunca fue perfecta, ni en el siglo xvi, manchada por la miseria y la intolerancia; ni en el xVIII, demasiado afrancesada, ni nunca. Concebimos una España en perpetua y vital renovación; en la que la tradición sea un compromiso y un estímulo, no una lápida o un sudario. Pero queremos que sea España; y si nos dan a escoger entre España, con unidad, con identidad y con honra, y cualquier otra cosa, diremos que lo nues- 
tro es España y que lo otro no nos sirve, porque será bueno para otros, pero a costa de España.

Esto supuesto, me propongo examinar, en el tiempo necesariamente limitado de un discurso, los siguientes cuatro puntos:

1. La Constitución y su posible reforma.

2. Las fuerzas políticas y su eventual remodelación.

3. La cultura cívica y el problema de los medios de información.

4. Una visión de la economía y de las relaciones sociales.

\section{La Constitución española de 1978 y su posible reforma}

No me voy a extender sobre los complejos problemas de lo que es y debe ser una Constitución y de cómo se ha llegado a la solución que hoy tenemos. Hay dos ideas de la Constitución: una, la británica, que la concibe como un conjunto dinámico de instituciones y de usos, que se va haciendo históricamente, como «un camino que anda»; otra, la francesa, como un texto escrito, que de vez en cuando se redacta en su totalidad por una asamblea constituyente. España, que ha tenido, de Jovellanos y Martínez Marina a Cánovas del Castillo y Gil y Robles (Enrique), grandes teóricos del primer punto de vista, ha aplicado generalmente el segundo, con poca fortuna, como todos sabemos. Nuestra larga historia constitucional es la de los fracasos de $1812,1820,1834,1836,1846,1851,1869,1873$ y 1931. Sólo han logrado alguna duración y estabilidad dos períodos: la Restauración, cuya Constitución de 1876 fue redactada por Cánovas, con un grupo de notables, y la era de las Leyes Fundamentales de Franco.

La Constitución vigente de 1978 ha vuelto a la vieja utopía de querer resolverlo y reformarlo todo a la vez; adolece de falta de realismo en varias de sus partes; los mismos que la hicieron la están forzando a menudo, en temas capitales como la redacción apresurada y ambigua de los Estatutos y el abuso de los decretos-leyes; está creando una desilusión creciente, por el retraso inevitable en el desarrollo legislativo y administrativo de sus numerosos y ambiciosos proyectos, $\mathrm{y}$, sobre todo, porque entretanto se van deteriorando la seguridad interior y exterior, la estabilidad económica y el empleo, la ley, el orden y el principio de autoridad.

Pues bien, nuestra clara posición en cuanto a la Constitución no ha variado. Primero, intentamos que la reforma política se hiciera por la otra vía, la de las reformas graduales y progresivas (como lo había intentado ya el primer Gobierno de la Monarquía). Rechazada esta idea, intentamos que el texto constitucional fuera más breve y más preciso (como, por ejemplo, la vigente Constitución francesa o la norteamericana), y no un largo código de todo lo divino y lo humano (como las Constituciones de Portugal y de Grecia).

Rechazadas ambas propuestas (que yo reiteré en la primera reunión de la Ponencia constitucional, en agosto de 1977), contribuimos de buena fe en los trabajos constitucionales (en la Ponencia, la Comisión y el Pleno del Congreso constituyente) a que el texto definitivo fuera lo mejor posible, y la verdad es que el segundo que salió de la Ponencia (tras el estudio de todas las enmiendas) era menos malo que lo que al fin prevaleció, tras los famosos «consensos». Aun así, estimamos (mayoritariamente) que era mejor hacer lo que hicimos: votar favorablemente el conjunto de la Constitución (gran parte de cuyos artículos eran aceptables) y mantener reservas, con propósito de la enmienda, en unas cuantas materias, respecto de las cuales anunciamos, en la explicación de voto, que en su día plantearíamos las correspondientes propuestas de reforma constitucional.

Estos puntos de desacuerdo no eran muchos, pero sí importantes. Se referían al artículo 2. (la funesta mención de las nacionalidades) y diversas disposiciones del 
ambiguo Título VIII, que desarrolla las autonomías; al sistema electoral, equivocadamente ratificado por la Constitución, que hace obligatorio el sistema proporcional, en vez de dejar esta cuestión a la Ley Electoral; a algunos artículos que afectan a instituciones fundamentales y a la moral cristiana (familia y educación, principalmente); $y$, en fin, a la antidemocrática restricción que los partidos han impuesto (reduciendo a muy poco la democracia semidirecta a través del referéndum y la iniciativa popular) al control popular del proceso político.

Hoy nuestra posición sigue siendo la misma, y será inútil todo intento de tergiversarla. No estamos, en primer lugar, por la destrucción de la Constitución; no creemos en actitudes puramente negativas ni de enfrentamiento entre los españoles; creemos que la Constitución es mejorable y perfectible, y le señalamos dos defectos graves, a los que se reducen los antes apuntados: los riesgos que tiene para la unidad política de España, para su identidad moral y cultural y para un Estado fuerte $\mathrm{y}$ eficente, $\mathrm{y}$ por otra parte, un sistema representativo excesivamente partitocrático.

Nuestra actitud no es, pues, ni de ruptura constitucional ni de aceptación resignada de las partes que rechazamos; es de reforma. Recordemos al respecto el ejemplo francés de 1958; en una grave crisis del país vecino, con Argelia perdida, con el Ejército sublevado, en plena crisis económica y social, De Gaulle ni cambió el régimen ni quiso imitar a Napoleón, a Boulanger, ni a Petain: reformó la Constitución (por vías legales), cambió el sistema electoral (pasando del proporcional al actual, mayoritario por distritos y a dos vueltas) y logró una nueva mayoría. Su sistema, plenamente democrático, ha funcionado con él, con Pompidou y con Giscard; ha superado crisis como la OAS y mayo de 1968; y ha vuelto a hacer de Francia un país grande, rico y fuerte. Italia, que continúa con instituciones parecidas a las que los franceses se dieron en 1946, sigue dividida y débil, aunque ahora empieza a pensar en la reforma constitucional.

Somos, pues, reformistas de la Constitución para hacerla viable, para que ella sola no se rompa como las anteriores. $Y$ esto lo mantendremos, una vez más, frente a los rupturistas, de uno $\mathrm{u}$ otro signo, o los conformistas que esperan con fatalismo a que las cosas acaben de estropearse.

Sería innecesario, pero ciertos comentarios de mala fe me obligan a aclarar que pongo la Constitución francesa de 1958 (ciertamente, la mejor que ha tenido la Francia contemporánea y una de las mejores de la Europa actual) como un ejemplo y no como un modelo. Ni las Constituciones se pueden copiar ni nadie sensato puede desear replantear la cuestión de la forma del Estado. El fracaso de las dos Repúblicas confirma que la Monarquía forma parte de la constitución histórica de España, y también sabemos que la secular institución puede ser dinámicamente puesta al día, al servicio de España, precisamente en sus problemas de hoy y de mañana.

\section{Las fuerzas políticas: su consolidación y remodelación}

Una Constitución es como un campo de juego; si el campo es malo, si está siempre embarrado, si tiene cuestas o grietas o sopla en él demasiado el viento, no se puede jugar un buen partido de fútbol. Pero dado que el campo sea bueno, la calidad del fútbol la hacen, más que Ia bella arquitectura de las fachadas o el acertado sistema de aparcamientos o jardines, los equipos buenos o malos.

Ya vimos que la Constitución no es ya el mejor de los campos posibles; pero los equipos que están jugando, los partidos políticos españoles actuales, son todavía más flojos e improvisados.

Un sistema de partidos políticos se define por los siguientes elementos:

a) El margen de influencia que los partidos tienen sobre el proceso político. 
b) El numero y tipo de los partidos que efectivamente participan en la lucha política.

c) Las relaciones que tienen unos con otros y la imagen que de las mismas llega al público.

En cuanto al primer punto, estimamos que hoy los partidos tienen una influencia excesiva en todas las fases del proceso político, y que por lo mismo nuestro sistema de partidos incide gravemente en el defecto de la partitocracia. Los partidos deben promover ideas, defender programas, proponer planes y candidatos, alertar y movilizar a la opinión. Pero hoy en España hacen muy poco de todo esto, y, en cambio, se dedican al control total de las listas electorales, al disfrute de amplias subvenciones estatales y de toda clase de prebendas, y todo ello con notoria falta de control constitucional y social.

Por lo que toca al segundo punto, la dolencia es la partiditis: hay demasiados partidos, poco claros en sus ideas y posiciones, y con tendencia a aumentar, como vemos en las noticias de cada día; tema que desgraciadamente puede agravarse en las elecciones a los Parlamentos regionales; recordemos, al respecto, que hay Constituciones (como la portuguesa) que prohíben los partidos de base regional, y que, incluso en países federales, como Estados Unidos o Alemania, los partidos tienen todos ámbito nacional.

En tercer lugar, el modo racional y normal de relacionarse los partidos, que es la dialéctica Gobierno-oposición, en España ha sido totalmente falseada y reemplazada por la ambigua del llamado consenso. En Inglaterra, en Estados Unidos, en Alemania, un partido gobierna y otro critica; en Francia, hay más de dos partidos, pero unos están en la mayoría que gobierna y otros en la minotía que hace oposición; en España, no: un Gobierno UCD pacta hoy con uno, mañana con otro, y a veces cambia tan rápidamente de pareja, que (como ocurrió en una reciente sesión del Congreso de los Diputados) cuando un orador está terminando un discurso diciendo que no a una proposición de ley, llegan por teléfono instrucciones cambiadas y UCD vota que sí.

Por otra parte, los señores Carrillo y González han dejado claro que apoyan al señor Suárez, cosa que sería inconcebible en cualquier país de sistema parlamentario normal. El resultado es que no hay Gobierno ni oposición; que la gente no sabe a qué atenerse sobre lo que representa cada uno; que a nadie le interesan ya los debates parlamentarios y las votaciones; y que ha llegado a inventarse la increíble expresión de «mayoría mecánica» para designar una mayoría sistemática que vota de modo coherente. En tan extraño sistema pasa como con el tero, el pájaro pampero de quien decía Martín Fierro que ponía los huevos en un sitio y daba las voces en otro. Se dice una cosa en el Congreso, otra en el mitin electoral, otra en el club de moda, y la confusión es completa.

El pueblo entonces, comprensiblemente, se abstiene, y piensa que no se trata de gobernar ni de criticar, sino de conservar la tajada.

Todos los planes para mejorar el futuro de nuestra España pasan, en lo político, por una remodelación del sistema de partidos. Esto quiere decir, a mi juicio:

a) Creación de fuerzas nacionales sólidas y responsables, con proyección y responsabilidad regional, pero evitando la proliferación de los grupos localistas y acabando con el falso mito del «sucursalismo», que no ha perjudicado a la izquierda, pero sí ha confundido y dividido a los grupos antimarxistas, como se ha visto en Cataluña.

b) Eliminación, en cumplimiento de la Constitución y de la ley, de las fuerzas revolucionarias y. separatistas, que usan la violencia para sus objetivos.

Hay que recordar que en todo hay un listón básico, una frontera clave, y que así ocurre también en los espacios políticos, nos guste o no. En la derecha hay una línea insalvable: que es la aceptación de la democracia y la renuncia a la violencia 
para imponer nuestras propias convicciones. En la izquierda, análogamente, hay otra línea divisoria: que es el recbazo de la idea de revolución entendida como transformación global del orden de cosas existentes, que es la línea que separa en Europa a la socialdemocracia de los partidos comunistas. En el terreno del regionalismo, la línea divisoria pasa por el separatismo frente a las actitudes foralistas o descentralizadoras.

Veamos ahora lo que tenemos en España. Subsisten grupos marxistas-leninistas, como el Partido Comunista (reconstituido), que utiliza métodos tertoristas a través de instrumentos como el GRAPO. El Partido Comunista Español ha adoptado el ropaje eurocomunista y ha quitado de su definición la palabra «leninista», pero sigue siendo un partido revolucionario, marxista y clasista que utiliza Comisiones Obreras y a múltiples otras asociaciones paralelas para una agitación permanente contra el sistema económico y social y que aspira a un cambio total del sistema político por la infiltración, la guerra cultural y otros procedimientos.

El PSOE ha hecho un interesante avance en la buena dirección, la socialdemocracia; pero, a diferencia de los demás partidos socialistas europeos, sigue anclado en la idea de partidos de clases y con una política exterior que no difiere en nada de la del Partido Comunista, incluso en la oposición a la NÂTO.

UCD no necesita mayores comentarios; a la vista están sus dos etapas de gobierno; su falta de principios, con espectáculos increíbles como esas intervenciones parlamentarias en las que se defiende el no y luego se vota el sí por un pacto telefónico de última hora. Nadie puede esperar del partido oficialista otra cosa que un continuado Gobierno por concesión, hasta que se acabe el capital y las rentas de las que hoy irresponsablemente se está tirando sin tasa.

Pues bien, ahora bablemos de nosotros. Es claro que nosotros, siendo como somos, partidarios del progreso y de la justicia social, no somos la izquierda; rechazamos el materialismo marxista, la indefinición en política exterior y de defensa, el confundir el progresismo con la permisividad, la falta de respeto a la tradición religiosa y moral de nuestro pueblo.

Es igualmente claro que siendo profunda $y$ auténticamente regionalistas, entusiastas de nuestras patrias chicas y de una España en su espléndida variedad, nosotros rechazamos los planteamientos nacionalistas por falsos y contrarios a la verdadera historia de España; las tesis racistas, por ridículas y anticristianas; los federalismos, por divisores y debilitadores del Estado que ha de defendernos a todos, y encarecedores de la administración y los servicios; y no hace falta decir que los separatismos por traidores y suicidas. Respetamos el foralismo, de buena solera hispánica; y aceptamos los Estatutos en la medida en que sirvan para una mejor integración de España.

Nosotros no somos tampoco un centro-izquierda, débil en los principios, oportunista en las actitudes, cambiante según la moda.

¿Qué somos, pues, nosotros? ¿Qué es y qué quiere ser Alianza Popular? Yo creo que somos la fuerza política que intenta (y lo logrará, si luchamos con tenacidad y organización) unir a los muchos españoles que queremos la unidad de España sin discusión; que rechazamos el marxismo con plena convicción de que el materialismo es lo más contrario a la esencia de nuestra Patria; que queremos una moral pública bien defendida; que defendemos las grandes instituciones sociales, como la familia, la Iglesia, las Fuerzas Armadas y de Orden Público, las escuelas públicas y privadas, etc.; que queremos progreso en todos los frentes de la vida nacional, pero dentro del orden y el respeto a la ley; y que, en fin, queremos todo eso por vías pacíficas, ciudadanas y democráticas. Esto no es nada utópico, puesto que existe en Inglaterra, como Partido Conservador; en Francia, como mayoría presidencial; en Estados Unidos, como Partido Republicano, y así sucesivamente.

Pues bien: sé que toco uno de los temas más polémicos de nuestra terminología 
política. Yo quisiera que no nos enzarzáramos en cuestiones nominalistas, y que estando como estamos de acuerdo en los principios, en los objetivos y en las actitudes, no nos dividamos (para gusto y ventaja de nuestros enemigos) en posicionamientos siempre relativos. Nosotros asumimos por supuesto los planteamientos espirituales, que son los válidos, de una derecha popular y para todos; rechazamos cualquier aspecto de represión o de privilegio o de oligarquía. Por eso somos lo que somos y seremos lo que debemos ser, y podemos dirigirnos a la mayoría natural del país, a la parte mayor y más sana de la sociedad, como decían los antiguos. Hoy desunida, desorientada, desencantada; que estuvo ahí, como casi un ochenta por ciento del cuerpo electoral, en el primer referéndum de la transición, en 1976, cuando la izquierda y los nacionalistas pidieron la abstención: tremenda responsabilidad por cierto la de aquellos que dejaron que se perdiera aquella ocasión magnífica y que aquella mayoría natural se confundiera y se desuniera. Esa es precisamente la explicación más importante de la creciente y preocupante abstención ciudadana, que es la de los que se sienten engañados, defraudados e incluso traicionados.

Nosotros tenemos que aspirar a recoger de nuevo a esa gran fuerza que está ahí; en un verdadero populismo, como el que sugiere nuestro mismo nombre, el de Alianza Popular. Ni podemos renunciar a él ni actuar de modo incongruente con esa idea: en la que quepa todo el pueblo, toda la sociedad real; que no se estrecha de modo que un aldeano, o un trabajador, o un estudiante, no se pueden encontrar representados. Sólo si hacemos esto, de buena fe, y lo hacemos bien, podremos llegar a ser un gran partido mayoritario, como España necesita.

Deseo, como el que más, una unión politica lo más amplia posible y basada en una organización eficaz de ese amplísimo sector, pero no puedo aceptarlo más que dentro de esquemas constitucionales (reformistas) y democráticos (de democracia fuerte). Así es como se han ganado las elecciones en Inglaterra y en Francia, y es como las podremos ganar nosotros si mantenemos la jugada, porque el tiempo nos está dando en todo la razón. Lo que no podemos es aceptar dudas sobre el principio claro de que no buscamos soluciones de otro tipo. Cuantos coincidan con este planteamiento serán bienvenidos; nosotros estamos dispuestos a cualquier sacrificio para lograrlo, y ya hemos demostrado nuestra auténtica disposición para hacerlo, sin más límites que los indicados. Lo que sí pedimos, antes de cualquier unión o acuerdo, es la aceptación expresa, por parte de todos, hacia dentro y hacia fuera, del principio democrático, del pluralismo político y de las libertades universales, tan necesitadas de apoyos inequívocos.

Tampoco debe olvidarse ( $\mathrm{y}$ bien lo sabemos por nuestra propia experiencia) que toda unión eficaz supone un sistema razonable y justo de pesas y medidas, de lo que realmente aporta cada uno, y algo nos ha enseñado la experiencia.

Conste, pues, nuestro respeto y simpatía a cuantos defienden a España; nuestra mejor disposición al diálogo, sin reservas ni condiciones; nuestra decisión de ir a cuantas uniones, colaboraciones y acuerdos nos permitan acercarnos a esa integración de la gran mayoría de nuestra sociedad. Lo que no podemos es entregar lo que es nuestro a otros, que pretenden administrarlo mejor; ni aceptar infiltraciones o submarinos que intenten paralizarnos o neutralizarnos. Nosotros somos nosotros, y lo seguiremos siendo, al servicio de España.

$\mathrm{Y}$ hoy tenemos una oportunidad real, como lo revela el movimiento de las ideas y las tendencias electorales en otros países de Europa. No hay oportunidad, a mi juicio, para los planteamientos integristas que se empeñen, desde una extrema derecha, en hacer una España para ellos solos, y para los métodos que intenten imponer una solución drástica, son métodos no democráticos. No la hay tampoco, a mi juicio, para una vieja derecsa, que está muerta en toda Europa; que se limite planteamientos capitalistas puros en lo económico, y a soluciones fuera del contexto del mundo actual, en el que los Estados medios ya no pueden resolver solos ni 
su defensa ni su política económica, ni tienen colonias, ni pueden moverse fuera del mundo que realmente existe. Hace falta una solución nueva: que está ahí, como lo demuestran, repito, las nuevas corrientes de pensamiento y los últimos resultados electorales.

En las ideas puede decirse que desde 1968 (el mayo francés y el golpe de Praga) el marxismo se bate en retirada en Europa; nadie puede ya creer que la revolución vaya a resolver los problemas de nuestros países. Los «nuevos filósofos» primero, la «nueva derecha» después, son los primeros pasos prometedores de que están apareciendo nuevas corrientes de pensamiento, en las cuales se podrá apoyar sin complejos una nueva vía de enfocar los problemas humanos, educativos, económicos, sociales y políticos. No me gusta mezclar los temas religiosos con los políticos; no somos un partido confesional, aunque tengamos una clara mayoría de católicos practicantes; no jugaremos nunca a servirnos de la Iglesia, sino a servirla, desde nuestra libre opción ciudadana. Pero a la vista está ese acontecimiento trascendental que está ocurriendo también en la Iglesia católica, con nuestro Papa, Juan Pablo II, que ha logrado una síntesis perfecta del tesoro de la tradición y de la asunción del mundo moderno y de la libertad sin dudas ni vacilaciones. La mayoría está con él, a pesar de que dice cosas que suponen esfuerzo, sacrificio, entrega; y no está ni con los sofisticados intérpretes que predicaban el fin de los dogmas y de la moral tradicional, ni tampoco con los reaccionarios que se empeñan en volver al «Syllabus» o en desconocer que la Iglesia ha de ser la primera en promover los derechos humanos y la justicia social.

$Y$ el veredicto de las urnas está igualmente cambiando de signo: ni en Francia, en 1978, ni en Inglaterra, en 1979, ni más recientemente en Suecia y Noruega, y esperamos que tampoco en Alemania el año próximo, se han producido desplazamientos a la izquierda, sino todo lo contrario. La democracia puede dar el triunfo a fuerzas a la vez nacionales y democráticas, conservadoras y reformistas. Lo que hace falta es trabajar para merecerlo. 